International Electronic Journal of Geometry

Volume 7 No. 1 PP. 26-35 (2014) (CIEJG

\title{
SOME RELATIONS BETWEEN NORMAL AND RECTIFYING CURVES IN MINKOWSKI SPACE-TIME
}

\author{
KAZIM İLARSLAN AND EMILIJA NEŠOVIĆ \\ Dedicated to memory of Proffessor Franki Dillen
}

\begin{abstract}
In this paper, we firstly give the necessary and sufficient conditions for null, pseudo null and partially null curves in Minkowski space-time to be normal curves. We prove that the null, pseudo null and partially null normal curves have a common property that their orthogonal projection onto non-degenerate hyperplane of $E_{1}^{4}$ or onto lightlike 2-plane of $E_{1}^{4}$ is the corresponding rectifying curve. Finally, we give some examples of such curves in $E_{1}^{4}$.
\end{abstract}

\section{InTRODUCTION.}

In the Euclidean space $\mathbb{E}^{3}$ there exist three classes of curves, so-called rectifying, normal and osculating curves satisfying Cesaro's fixed point condition ([8]) meaning that rectifying, normal and osculating planes of such curves always contain a particular point. If all normal or osculating planes of a curve in $\mathbb{E}^{3}$ pass through a particular point, then the curve is spherical or planar, respectively. It is also known that if all rectifying planes of a non-planar curve in $\mathbb{E}^{3}$ pass through a particular point, then the ratio of its torsion and curvature is a non-constant linear function ([3]). Some characterizations of rectifying curves in Minkowski 3-space $E_{1}^{3}$ are given in [6]. In particular, there exists a simple relationship between rectifying curves and Darboux vectors (centrodes), which play some important roles in mechanics, kinematics as well as in differential geometry in defining the curves of constant precession $([4])$.

Normal curves in Minkowski space-time $\mathrm{E}_{1}^{4}$ are defined in [5] as the space curves whose position vector (with respect to some chosen origin) always lies in its normal space $T^{\perp}$, which represents the orthogonal complement of the tangent vector field of the curve. Spacelike and timelike normal curves in $\mathrm{E}_{1}^{4}$, whose Frenet frame contains only non-null vector fields, are characterized in [5]. Such curves always lie in some hyperquadric in $\mathrm{E}_{1}^{4}$.

In this paper, we firstly give the necessary and sufficient conditions for null, pseudo null and partially null curves in Minkowski space-time to be normal curves.

2010 Mathematics Subject Classification. Primary 53C50; Secondary 53C40.

Key words and phrases. rectifying curve, normal curve, Minkowski space-time. 
It is a quite interesting problem to investigate if there exist some geometrical relations between null, pseudo null and partially null normal curves and rectifying curves in Minkowski space-time. In this paper we prove that the null, pseudo null and partially null normal curves have a common property that their orthogonal projection onto non-degenerate hyperplane of $E_{1}^{4}$ or onto lightlike 2-plane of $E_{1}^{4}$ is the corresponding rectifying curve. Finally, we give some examples of such null, pseudo null and partially null normal curves in $E_{1}^{4}$.

\section{Preliminaries.}

The Minkowski space-time $E_{1}^{4}$ is the Euclidean 4 -space $E^{4}$ equipped with indefinite flat metric given by

$$
g=-d x_{1}^{2}+d x_{2}^{2}+d x_{3}^{2}+d x_{4}^{2},
$$

where $\left(x_{1}, x_{2}, x_{3}, x_{4}\right)$ is a rectangular coordinate system of $E_{1}^{4}$. Recall that a vector $v \in E_{1}^{4} \backslash\{0\}$ can be spacelike if $g(v, v)>0$, timelike if $g(v, v)<0$ and null (lightlike) if $g(v, v)=0$. In particular, the vector $v=0$ is a spacelike. The norm of a vector $v$ is given by $\|v\|=\sqrt{|g(v, v)|}$. Two vectors $v$ and $w$ are said to be orthogonal, if $g(v, w)=0$. An arbitrary curve $\alpha(s)$ in $E_{1}^{4}$, can locally be spacelike, timelike or null (lightlike), if all its velocity vectors $\alpha^{\prime}(s)$ are respectively spacelike, timelike or null ([7]). Spacelike curve in $E_{1}^{3}$ or $E_{1}^{4}$ is called respectively pseudo null or partially null curve, if its principal normal or first binormal vector is null ([2]).

A null curve $\alpha$ is parameterized by pseudo-arc $s$ if $g\left(\alpha^{\prime \prime}(s), \alpha^{\prime \prime}(s)\right)=1([1])$. A non-null curve $\alpha$ has unit speed, if $g\left(\alpha^{\prime}(s), \alpha^{\prime}(s)\right)= \pm 1$.

Recall that the pseudosphere, pseudohyperbolic space and nullcone are the hyperquadrics in $E_{1}^{4}$ respectively defined by ([7])

$$
\begin{gathered}
S_{1}^{3}(r)=\left\{X \in E_{1}^{4}: g(X, X)=r^{2}, r \in R_{0}^{+}\right\}, \\
H_{0}^{3}(r)=\left\{X \in E_{1}^{4}: g(X, X)=-r^{2}, r \in R_{0}^{+}\right\}, \\
C^{3}(O)=\left\{X \in E_{1}^{4}: g(X, X)=0, X \neq 0\right\} .
\end{gathered}
$$

Let $\left\{T, N, B_{1}, B_{2}\right\}$ be the moving Frenet frame along a curve $\alpha$ in $E_{1}^{4}$, consisting of the tangent, the principal normal, the first binormal and the second binormal vector field respectively. Depending on the causal character of $\alpha$, the Frenet equations have the following forms.

Case I. If $\alpha$ is a null curve, the Frenet equations are given by $([1,10])$

$$
\left[\begin{array}{l}
T^{\prime} \\
N^{\prime} \\
B_{1}^{\prime} \\
B_{2}^{\prime}
\end{array}\right]=\left[\begin{array}{cccc}
0 & \kappa_{1} & 0 & 0 \\
\kappa_{2} & 0 & -\kappa_{1} & 0 \\
0 & -\kappa_{2} & 0 & \kappa_{3} \\
-\kappa_{3} & 0 & 0 & 0
\end{array}\right]\left[\begin{array}{c}
T \\
N \\
B_{1} \\
B_{2}
\end{array}\right]
$$

where the first curvature $\kappa_{1}(s)=0$, if $\alpha(s)$ is straight line, or $\kappa_{1}(s)=1$ in all other cases. Therefore, such curve has two curvatures $\kappa_{2}(s)$ and $\kappa_{3}(s)$ and the following equations hold:

$$
\begin{gathered}
g(T, T)=g\left(B_{1}, B_{1}\right)=0, \quad g(N, N)=g\left(B_{2}, B_{2}\right)=1 \\
g(T, N)=g\left(T, B_{2}\right)=g\left(N, B_{1}\right)=g\left(N, B_{2}\right)=g\left(B_{1}, B_{2}\right)=0, \quad g\left(T, B_{1}\right)=1 .
\end{gathered}
$$


Case II. If $\alpha$ is pseudo null curve, the Frenet formulas are $([2,10])$

$$
\left[\begin{array}{l}
T^{\prime} \\
N^{\prime} \\
B_{1}^{\prime} \\
B_{2}^{\prime}
\end{array}\right]=\left[\begin{array}{cccc}
0 & \kappa_{1} & 0 & 0 \\
0 & 0 & \kappa_{2} & 0 \\
0 & \kappa_{3} & 0 & -\kappa_{2} \\
-\kappa_{1} & 0 & -\kappa_{3} & 0
\end{array}\right]\left[\begin{array}{c}
T \\
N \\
B_{1} \\
B_{2}
\end{array}\right]
$$

where the first curvature $\kappa_{1}(s)=0$, if $\alpha$ is straight line, or $\kappa_{1}(s)=1$ in all other cases. Such curve has two curvatures $\kappa_{2}(s)$ and $\kappa_{3}(s)$ and the following conditions are satisfied:

$$
\begin{gathered}
g(T, T)=g\left(B_{1}, B_{1}\right)=1, \quad g(N, N)=g\left(B_{2}, B_{2}\right)=0 \\
g(T, N)=g\left(T, B_{1}\right)=g\left(T, B_{2}\right)=g\left(N, B_{1}\right)=g\left(B_{1}, B_{2}\right)=0, \quad g\left(N, B_{2}\right)=1 .
\end{gathered}
$$

Case III. If $\alpha$ is partially null curve, the Frenet formulas read $([2,10])$

$$
\left[\begin{array}{l}
T^{\prime} \\
N^{\prime} \\
B_{1}^{\prime} \\
B_{2}^{\prime}
\end{array}\right]=\left[\begin{array}{cccc}
0 & \kappa_{1} & 0 & 0 \\
-\kappa_{1} & 0 & \kappa_{2} & 0 \\
0 & 0 & \kappa_{3} & 0 \\
0 & -\kappa_{2} & 0 & -\kappa_{3}
\end{array}\right]\left[\begin{array}{c}
T \\
N \\
B_{1} \\
B_{2}
\end{array}\right]
$$

where the third curvature $\kappa_{3}(s)=0$ for each $s$. Such curve has two curvatures $\kappa_{1}(s)$ and $\kappa_{2}(s)$ and lies fully in a lightlike hyperplane of $E_{1}^{4}$. In particular, the following equations hold:

$$
\begin{gathered}
g(T, T)=g(N, N)=1, \quad g\left(B_{1}, B_{1}\right)=g\left(B_{2}, B_{2}\right)=0 \\
g(T, N)=g\left(T, B_{1}\right)=g\left(T, B_{2}\right)=g\left(N, B_{1}\right)=g\left(N, B_{2}\right)=0, \quad g\left(B_{1}, B_{2}\right)=1 .
\end{gathered}
$$

Recall that a rectifying curve in $E_{1}^{3}$ is defined in [6] as a space curve whose the position vector (with respect to some chosen origin) always lies in its rectifying plane

$$
N^{\perp}=\left\{W \in E_{1}^{3} \mid g(W, N)=0\right\} .
$$

In particular, the normal curve in $E_{1}^{4}$ is defined in [5] as a curve whose the position vector (with respect to some chosen origin) always lies in its normal space given by

$$
T^{\perp}=\left\{W \in E_{1}^{4} \mid g(W, T)=0\right\} .
$$

Consequently, the position vector of null, pseudo null and partially null normal curve in $E_{1}^{4}$, satisfies respectively the equations

$$
\begin{gathered}
\alpha(s)=a(s) T(s)+b(s) N(s)+c(s) B_{2}(s), \\
\alpha(s)=a(s) N(s)+b(s) B_{1}(s)+c(s) B_{2}(s), \\
\alpha(s)=a(s) N(s)+b(s) B_{1}(s),
\end{gathered}
$$

for some differentiable functions $a(s), b(s)$ and $c(s)$. 


\section{Null, PSEUdo NUll AND PARTIALly NULl NORMAL CURVES IN $E_{1}^{4}$}

In this section, we firstly give the necessary and sufficient conditions for null, pseudo null and partially null curves in $E_{1}^{4}$ to be normal curves. One of the sufficient conditions is that such curves (with the first and the second curvature different from zero) always lie in some hyperquardic of the ambient space. It can be easily verified that every null straight line in $E_{1}^{4}$ is a normal curve. For the null normal curves in $E_{1}^{4}$ with non-zero first curvature we have the following theorem.

Theorem 3.1. Let $\alpha(s)$ be a null curve in $E_{1}^{4}$ parameterized by pseudo-arc $s$ and with curvatures $k_{1}(s)=1, k_{2}(s) \neq 0, k_{3}(s) \neq 0$. Then $\alpha$ is a normal curve if and only if one of the following statements hold:

(i) $\alpha$ lies in pseudosphere $S_{1}^{3}(r), r \in R_{0}^{+}$;

(ii) the third curvature $\kappa_{3}(s)$ is non-zero constant;

(iii) the second binormal component of the position vector $\alpha$ is non-zero constant, i.e. $g\left(\alpha, B_{2}\right)=c_{0}, c_{0} \in R_{0}$.

Proof. First assume that $\alpha$ is a normal curve. By taking the derivative of the relation (2.4) with respect to $s$ and applying (2.1), we obtain the system of equations

$$
a^{\prime}(s)+b(s) \kappa_{2}(s)-c(s) \kappa_{3}(s)=1, a(s)+b^{\prime}(s)=0, b(s)=0, c^{\prime}(s)=0 .
$$

It follows that

$$
a(s)=0, \quad b(s)=0, \quad c(s)=c_{0}, \quad k_{3}(s)=-1 / c_{0}, \quad c_{0} \in R_{0} .
$$

Therefore, statement (ii) holds. Substituting (3.1) in (2.4), we easily obtain that statements (i) and (iii) hold.

Conversely, assume that statement (i) holds. Differentiating the equation $g(\alpha, \alpha)=$ $r^{2}$ with respect to $s$, we find $g(\alpha, T)=0$ which means that $\alpha$ is the normal curve. If statement (ii) holds, putting $k_{3}(s)=-1 / c_{0}, c_{0} \in R_{0}$ and applying (2.1) we obtain $d / d s\left[\alpha(s)-c_{0} B_{2}(s)\right]=0$. Thus $\alpha$ is congruent to a normal curve. If statement (iii) holds, differentiating the equation $g\left(\alpha, B_{2}\right)=c_{0}$ with respect to $s$, we easily obtain that $\alpha$ is a normal curve which completes the proof.

Theorem 3.2. Let $\alpha(s)$ be a unit speed pseudo null curve in $E_{1}^{4}$ with curvatures $k_{1}(s)=1, k_{2}(s) \neq 0$ and $k_{3}(s) \neq 0$. Then $\alpha$ is a normal curve if and only if one of the following statements hold:

(i) $\alpha$ lies in pseudosphere or pseudohyperbolic space in $E_{1}^{4}$;

(ii) the first binormal and the second binormal component of the position vector $\alpha$ are respectively given by $g\left(\alpha, B_{1}\right)=0, g\left(\alpha, B_{2}\right)=c, c \in R$.

Proof. First assume that $\alpha$ is a normal curve. Then its position vector satisfies relation (2.5). By taking the derivative of (2.5) with respect to $s$ and applying (2.2), we obtain the system of equations

$$
\begin{aligned}
& c(s)=-1, \quad c^{\prime}(s)-b(s) \kappa_{2}(s)=0, \\
& a(s) \kappa_{2}(s)+b^{\prime}(s)-c(s) \kappa_{3}(s)=0, \quad a^{\prime}(s)+b(s) \kappa_{3}(s)=0 .
\end{aligned}
$$

From the first and second equation of (3.2), we get $b(s)=0$. Therefore, the system of equations (3.2) reduces to the system

$$
a(s)=-\kappa_{3}(s) / \kappa_{2}(s), b(s)=0, c(s)=-1, a^{\prime}(s)=0 .
$$


From the first and the last equation of (3.3) we get $a(s)=a_{0} \in R_{0}$. Consequently, by using the last relation and (3.3), relation (2.5) becomes

$$
\alpha(s)=a_{0} N(s)-B_{2}(s), \quad a_{0} \in R_{0} .
$$

The last relation implies $g(\alpha, \alpha)=-2 a_{0}, g\left(\alpha, B_{1}\right)=0, g\left(\alpha, B_{2}\right)=a_{0}, a_{0} \in R_{0}$, which proves statements (i) and (ii).

Conversely, suppose that statement (i) holds. Differentiating the equation $g(\alpha, \alpha)=$ $c, c \in R$ with respect to $s$ we easily get that $\alpha$ is a normal curve. If statement (ii) holds, by taking the derivative of the equation $g\left(\alpha, B_{2}\right)=c$ with respect to $s$, it follows that $\alpha$ is a normal curve, which proves the theorem.

Remark 3.1. If pseudo null normal curve has the third curvature $\kappa_{3}(s)=0$, then its parameter equation reads $\alpha(s)=-B_{2}(s)$, so it lies in the lightcone $C^{3}(O)$.

The following theorem can be proved in a similar way as theorems 3.1 and 3.2., so we omit its proof.

Theorem 3.3. A unit speed partially null curve $\alpha(s)$ in $E_{1}^{4}$ with the curvatures $\kappa_{1}(s) \neq 0, \kappa_{2}(s) \neq 0$ and $\kappa_{3}(s)=0$ is a normal curve if and only if one of the following statements hold:

(i) the first curvature $\kappa_{1}(s)$ is non-zero constant;

(ii) $\alpha$ lies in a circular cylinder with null axis;

(iii) the principal normal and the first binormal component of the position vector $\alpha$ are respectively given by $g(\alpha, N)=a_{0}, a_{0} \in R_{0}, g\left(\alpha, B_{1}\right)=0$.

(iv) the first binormal and the second binormal component of the position vector $\alpha$ are respectively given by $g\left(\alpha, B_{1}\right)=0, g\left(\alpha, B_{2}\right)=-a_{0} \int_{0}^{s} \kappa_{2}(s) d s, a_{0} \in R_{0}$.

Recall that arbitrary curve in $E_{1}^{4}$ is called a helix or a $W$-curve, if all its curvature functions are constant. It is known that every pseudo null helix lies in some hyperquadric in $E_{1}^{4}([9])$.

Corollary 3.1. Every null, pseudo null and partially null helix in $E_{1}^{4}$ is a normal curve.

Next we obtain a simple relationship between null normal curves and non-null rectifying curves in Minkowski space-time.

Theorem 3.4. Let $\alpha$ be a null curve in $E_{1}^{4}$ and let $\beta$ be the orthogonal projection of $\alpha$ onto the spacelike (timelike) hyperplane $\Sigma$ of $E_{1}^{4}$. Then $\alpha$ is a normal curve if and only if $\beta$ is a spacelike (timelike) rectifying curve.

Proof. We give the proof when $\Sigma$ is a spacelike hyperplane. When $\Sigma$ is a timelike hyperplane, the proof is analogous.

Assume that $\alpha$ is a normal curve. The equation of $\alpha$ in terms of $\beta$ can be written as

$$
\alpha(s)=\beta(s)+\mu(s) V
$$

where $s$ is arclength parameter of $\beta, \mu(s)$ is a nonconstant differentiable function and $V \in \Sigma^{\perp}$ is a constant unit timelike vector. Taking the derivative of (3.4) with respect to $s$ and using the condition $g\left(\alpha^{\prime}(s), \alpha^{\prime}(s)\right)=0$ we get $g\left(\beta^{\prime}(s), \beta^{\prime}(s)\right)=$ $\mu^{\prime 2}(s)=1$. Up to a translations of $E_{1}^{4}$, we may take

$$
\mu(s)=s .
$$


From the condition $g\left(\alpha(s), \alpha^{\prime}(s)\right)=0$, we obtain

$$
g\left(\beta(s), \beta^{\prime}(s)\right)=\mu(s) \mu^{\prime}(s) .
$$

Differentiating the previous equation with respect to $s$ and using (3.5) we find $g\left(\beta(s), \beta^{\prime \prime}(s)\right)=0$, which means that $\beta$ is a spacelike rectifying curve.

Conversely, assume that the projected curve $\beta$ is a spacelike rectifying curve lying fully in the spacelike hyperplane $\Sigma$ of $E_{1}^{4}$. Then $\beta$ is given by ([3])

$$
\beta(t)=\frac{a}{\cos t} y(t), \quad a \in R_{0}^{+},
$$

where $y(t)$ is a unit speed spacelike curve lying in the unit sphere $S^{2}(1)$. Hence the equation of $\alpha$ can be written as

$$
\alpha(t)=\frac{a}{\cos t} y(t)+\mu(t) V, \quad a \in R_{0}^{+},
$$

where $\mu(t)$ is a nonconstant differentiable function and $V \in \Sigma^{\perp}$ is a constant unit timelike vector. By using the condition $g\left(\alpha^{\prime}(t), \alpha^{\prime}(t)\right)=0$, we obtain $\mu(t)=a \tan t$. Substituting this in (3.6), it follows that $g\left(\alpha(t), \alpha^{\prime}(t)\right)=0$, which means that $\alpha$ is a normal curve.

It can be verified that the orthogonal projection of a pseudo null normal curve $\alpha$ in $E_{1}^{4}$ onto non-degenerate hyperplane of $E_{1}^{4}$ is not necessarily a rectifying curve. However, the mentioned projection can be a rectifying curve under some additional conditions given in the next theorem.

Theorem 3.5. Let $\alpha$ be non-null normal curve in $E_{1}^{4}$ and $\beta$ a unit speed timelike orthogonal projection of $\alpha$ onto timelike hyperplane $\Sigma$ of $E_{1}^{4}$. Then $\alpha$ is pseudo null curve and holds

$$
g(\alpha(s), V)=\sqrt{s^{2}+a}, \quad a \in R_{0}^{-},
$$

where $s$ is arclength parameter of $\beta$ and $V \in \Sigma^{\perp}$ is a constant unit spacelike vector, if and only if $\beta$ is a rectifying curve with the first curvature

$$
k_{\beta}(s)=\frac{\sqrt{|a|}}{s^{2}+a}, \quad a \in R_{0}^{-} .
$$

Proof. First assume that $\alpha$ is pseudo null normal curve and holds $g(\alpha(s), V)=$ $\sqrt{s^{2}+a}, a \in R_{0}^{-}$. The equation of $\alpha$, in terms of its orthogonal projection $\beta$, can be written as

$$
\alpha(s)=\beta(s)+\mu(s) V,
$$

where $\mu(s)=g(\alpha(s), V)$. Differentiating the previous equation with respect to $s$ we obtain

$$
\alpha^{\prime}(s)=\beta^{\prime}(s)+\mu^{\prime}(s) V .
$$

Since $\alpha$ is a normal curve, it follows that $g\left(\alpha(s), \alpha^{\prime}(s)\right)=0$. Substituting (3.9) and (3.10) into the last equation, we get

$$
g\left(\beta(s), \beta^{\prime}(s)\right)+\mu(s) \mu^{\prime}(s)=0 .
$$

Differentiating the previous equation with respect to $s$ and using the conditions $g\left(\beta^{\prime}(s), \beta^{\prime}(s)\right)=-1, \mu(s)=\sqrt{s^{2}+c}, c \in R_{0}^{-}$, we obtain $g\left(\beta(s), N_{\beta}(s)\right)=0$. Hence $\beta$ is a rectifying curve. Denote by $t$ arclength parameter of $\alpha$. Since $\alpha(s)=\alpha(t(s))$, 
differentiating the last equation two times with respect to $s$ and using the condition $g\left(\alpha^{\prime \prime}(t), \alpha^{\prime \prime}(t)\right)=0$, we obtain

$$
g\left(\alpha^{\prime \prime}(s), \alpha^{\prime \prime}(s)\right)=t^{\prime \prime 2}(s) .
$$

In particular, differentiating relation (3.10) with respect to $s$ yields

$$
g\left(\alpha^{\prime \prime}(s), \alpha^{\prime \prime}(s)\right)=\kappa_{\beta}^{2}(s)+\mu^{\prime \prime 2}(s) .
$$

The arclength parameter $t$ of $\alpha$ is given by

$$
t(s)=\int_{0}^{s}\left\|\alpha^{\prime}(u)\right\| d u=\int_{0}^{s} \sqrt{\mu^{\prime 2}(u)-1} d u .
$$

Differentiating relation (3.13) two times with respect to $s$ and using (3.11) and (3.12) we obtain that the first curvature $\kappa_{\beta}$ is given by (3.8).

Conversely, assume that $\beta$ is a timelike rectifying curve with the first curvature given by (3.8). Then $\beta$ has parameter equation ([6])

$$
\beta(u)=\frac{a}{\sinh u} y(u), \quad a \in R_{0}^{+},
$$

where $y(u)$ is the unit speed spacelike curve lying in pseudohyperbolic space $H_{0}^{2}(1)$. The last equation in terms of arclength parameter $s$ of $\beta$, reads

$$
\beta(s)=y(s) \sqrt{s^{2}-a^{2}}, \quad a \in R_{0}^{+} .
$$

Substituting the last relation in (3.9) we get

$$
\alpha(s)=y(s) \sqrt{s^{2}-a^{2}}+\mu(s) V .
$$

Differentiating the previous equation with respect to $s$ we find

$$
\alpha^{\prime}(s)=y^{\prime}(s) \sqrt{s^{2}-a^{2}}+y(s) \frac{s}{\sqrt{s^{2}-a^{2}}}+\mu^{\prime}(s) V .
$$

Since $\alpha$ is a normal curve, there holds $g\left(\alpha(s), \alpha^{\prime}(s)\right)=0$. Substituting (3.14) and (3.21) into the last equation we find

$$
\mu(s)=g(\alpha(s), V)=\sqrt{s^{2}+d}, \quad d \in R,
$$

and therefore

$$
\mu^{\prime \prime}(s)=\frac{d}{\left(s^{2}+d\right)^{\frac{3}{2}}}, \quad d \in R .
$$

Differentiating relation (3.9) two times with respect to $s$ yields

$$
\alpha^{\prime \prime}(s)=\beta^{\prime \prime}(s)+\mu^{\prime \prime}(s) V .
$$

It follows that

$$
g\left(\alpha^{\prime \prime}(s), \alpha^{\prime \prime}(s)\right)=\kappa_{\beta}^{2}(s)+\mu^{\prime \prime 2}(s) .
$$

In particular, substituting (3.8) and (3.17) into the last equation, we find

$$
g\left(\alpha^{\prime \prime}(s), \alpha^{\prime \prime}(s)\right)=-\frac{a}{\left(s^{2}+a\right)^{2}}+\frac{d^{2}}{\left(s^{2}+d\right)^{3}}, \quad a \in R_{0}^{-}, d \in R .
$$

Without loss of generality, we may take $d=a$. Then the last equation becomes

$$
g\left(\alpha^{\prime \prime}(s), \alpha^{\prime \prime}(s)\right)=-\frac{a s^{2}}{\left(s^{2}+a\right)^{3}}, \quad a \in R_{0}^{-} .
$$

In particular, relation (3.16) becomes 


$$
\mu(s)=g(\alpha(s), V)=\sqrt{s^{2}+a}, \quad a \in R_{0}^{-} .
$$

Denote by $t$ arclength parameter of $\alpha$.Differentiating the equation $\alpha(s)=\alpha(t(s))$ two times with respect to $s$ we get $\alpha^{\prime \prime}(s)=\alpha^{\prime \prime}(t) t^{\prime 2}+\alpha^{\prime}(t) t^{\prime \prime}$. The last relation implies

$$
g\left(\alpha^{\prime \prime}(s), \alpha^{\prime \prime}(s)\right)=g\left(\alpha^{\prime \prime}(t), \alpha^{\prime \prime}(t)\right) t^{4}+g\left(\alpha^{\prime}(t), \alpha^{\prime}(t)\right) t^{\prime 2} .
$$

On the other hand, from (3.13) we have

$$
t^{\prime}=\sqrt{\mu^{\prime 2}-1}, \quad t^{\prime \prime}=\frac{\mu^{\prime} \mu^{\prime \prime}}{\sqrt{\mu^{\prime 2}-1}} .
$$

Substituting this into (3.20) we obtain

$$
g\left(\alpha^{\prime \prime}(s), \alpha^{\prime \prime}(s)\right)=\kappa_{\alpha}^{2}(t) g\left(N_{\alpha}(t), N_{\alpha}(t)\right)\left(\mu^{\prime 2}-1\right)^{2}+g\left(\alpha^{\prime}(t), \alpha^{\prime}(t)\right) \frac{\mu^{\prime 2} \mu^{\prime \prime 2}}{\mu^{\prime 2}-1} .
$$

By using (3.19), the last equation becomes

$$
g\left(\alpha^{\prime \prime}(s), \alpha^{\prime \prime}(s)\right)=\kappa_{\alpha}^{2}(t) g\left(N_{\alpha}(t), N_{\alpha}(t)\right) \frac{a^{2}}{\left(s^{2}+a\right)^{2}}-g\left(\alpha^{\prime}(t), \alpha^{\prime}(t)\right) \frac{a s^{2}}{\left(s^{2}+a\right)^{3}} .
$$

The last equation and (3.18) imply

$$
\frac{s^{2}}{s^{2}+a}\left(g\left(\alpha^{\prime}(t), \alpha^{\prime}(t)\right)-1\right)=a \kappa_{\alpha}^{2}(t) g\left(N_{\alpha}(t), N_{\alpha}(t)\right), \quad a \in R_{0}^{-} .
$$

By assumption, $\alpha$ is non-null curve, so $g\left(\alpha^{\prime}(t), \alpha^{\prime}(t)\right)= \pm 1$. If $g\left(\alpha^{\prime}(t), \alpha^{\prime}(t)\right)=$ -1 , relations $\alpha^{\prime}(s)=\beta^{\prime}(s)+\mu^{\prime}(s) V$ and $\alpha^{\prime}(s)=\alpha^{\prime}(t) t^{\prime}(s)$ imply

$$
g\left(\alpha^{\prime}(s), \alpha^{\prime}(s)\right)=-t^{\prime 2}(s)=g\left(\beta^{\prime}(s), \beta^{\prime}(s)\right)+\mu^{\prime 2}(s) .
$$

Relation (3.13) implies $t^{\prime 2}=\mu^{\prime 2}-1$. Substituting this into the last equation, we find $g\left(\beta^{\prime}(s), \beta^{\prime}(s)\right)=1-2 \mu^{\prime 2}(s)=-1$. It follows that $\mu(s)= \pm s+b, b \in R$, which is a contradiction with (3.19). Hence $g\left(\alpha^{\prime}(t), \alpha^{\prime}(t)\right)=1$, so relation (3.21) implies $g\left(N_{\alpha}, N_{\alpha}\right)=0$ which means that $\alpha$ is a pseudo null curve. This completes the proof of the theorem.

The relationship between partially null normal curves and rectifying curves is given in the last theorem.

Theorem 3.6. Let $\alpha$ be a partially null curve in $E_{1}^{4}$ and $\beta$ a spacelike orthogonal projection of $\alpha$ onto lightlike 2-plane of $E_{1}^{4}$. If $\alpha$ is a normal curve, then $\beta$ is a rectifying curve.

Proof. Assume that $\alpha$ is partially null normal curve lying in the lightlike hyperplane $\Omega$ of $E_{1}^{4}$. We may assume that up to isometries of $E_{1}^{4}, \Omega$ has the equation $x_{1}=x_{2}$. Then up to a parametrization, parameter equation of $\alpha$ reads

$$
\alpha(s)=\left(\alpha_{1}(s), \alpha_{1}(s), s, \sqrt{r^{2}-s^{2}}\right), \quad r \in R_{0}^{+},
$$

where $\alpha_{1}$ is some differentiable function. Moreover, the orthogonal projection of $\alpha$ onto lightlike 2-plane has the equation

$$
\beta(s)=\left(\alpha_{1}(s), \alpha_{1}(s), s, 0\right) .
$$

It follows that $g(\beta(s), \beta(s))=s^{2}$. Differentiating the last equation two times with respect to $s$, we get $g\left(\beta(s), N_{\beta}(s)\right)=0$, which means that $\beta$ is a rectifying curve. 


\section{Some EXAmples of NUll, PSEUdo Null AND PARTIALly NULl NORMAL CURVES IN $\mathrm{E}_{1}^{4}$}

In this section, we give some examples of null, pseudo null and partially normal curves in terms of their orthogonal projections onto non-degenerate hyperplanes and lightlike 2-plane of $E_{1}^{4}$. Denote by $\Sigma$ non-degenerate hyperplane of $E_{1}^{4}$.

Case (1) $\Sigma$ is spacelike. By theorem 3.4 the null normal curve $\alpha$ is given by

$$
\alpha(s)=\beta(s)+s V,
$$

where the rectifying curve $\beta(s)=\sqrt{s^{2}+c^{2}} y(s), c \in R_{0}^{+}$, is the unit speed orthogonal projection of $\alpha$ onto $\Sigma, y(s)$ is a spacelike curve lying in the unit sphere $S^{2}(1)$ and $V \in \Sigma^{\perp}$ is a constant unit timelike vector.

Case (2) $\Sigma$ is timelike. According to theorem 3.4, the null normal curve $\alpha$ has the equation

$$
\alpha(s)=\beta(s)+s V, \quad c \in R_{0}^{+},
$$

where the rectifying curve $\beta(s)=(c-s) \sqrt{\frac{s+c}{s-c}} y(s)$ is the unit speed orthogonal projection of $\alpha$ onto $\Sigma, y(s)$ is a spacelike curve lying in pseudohyperbolic space $H_{0}^{2}(1)$ and $V \in \Sigma^{\perp}$ is a constant unit spacelike vector.

According to theorem 3.5, parameter equation of pseudo null normal curve is given by

$$
\alpha(s)=\beta(s)+\sqrt{s^{2}+a} V, \quad a \in R_{0}^{-},
$$

where the rectifying curve $\beta(s)=(c-s) \sqrt{\frac{s+c}{s-c}} y(s), c \in R_{0}^{+}$, is the unit speed orthogonal projection of $\alpha$ onto timelike hyperplane $\Sigma, y(s)$ is a spacelike curve lying in pseudohyperbolic space $H_{0}^{2}(1)$ and $V \in \Sigma^{\perp}$ is a constant unit spacelike vector.

Finally, according to theorem 3.6, parameter equation of partially null normal curve can be written as

$$
\alpha(s)=\left(s^{2}, s^{2}, s, \sqrt{r^{2}-s^{2}}\right), \quad r \in R_{0}^{+},
$$

where the rectifying curve $\beta(s)=\left(s^{2}, s^{2}, s, 0\right)$ is the orthogonal projection of $\alpha$ onto lightlike 2-plane of $E_{1}^{4}$. Since $\kappa_{\beta}(s)=1$ and $\tau_{\beta}(s)=0$, the projected curve $\beta$ is a pseudo null circle.

\section{REFERENCES}

[1] Bonnor, W. B., Null curves in a Minkowski space-time, Tensor 20, 229-242, 1969.

[2] Bonnor, W. B., Curves with null normals in Minkowski space-time (A random walk in relativity and cosmology, Wiley Easten Limitid 33-47, 1985)

[3] Chen, B. Y., When does the position vector of a space curve always lie in its rectifying plane?, Amer. Math. Monthly 110, 147-152, 2003.

[4] Chen, B.Y. and Dillen F., Rectifying curves as centrodes and extremal curves, Bull. Inst. Math. Acad. Sinica 33(2), 77-90, 2005.

[5] İlarslan, K and Nešović, E., Spacelike and timelike normal curves in Minkowski space-time, Publ. Inst. Math. Belgrade 85 (99), 111-118, 2009.

[6] İlarslan, K., Nešović, E. and Petrović-Torgašev M., Some characterizations of rectifying curves in the Minkowski 3-space, Novi Sad J. Math. 33(2), 23-32, 2003.

[7] O'Neill, B., Semi-Riemannian geometry with applications to relativity (Academic Press, New York, 1983).

[8] Otsuki, T., Differential Geometry, (In Japanese, Asakura Publishing Co. Ltd., 1961).

[9] Petrović-Torgašev, M. and Šućurović E., W-curves in Minkowski space-time, Novi Sad J. Math. 32(2), 55-65, 2002. 
[10] Valrave, J., Curves and surfaces in Minkowski space (Doctoral thesis, K. U. Leuven, Fac. of Science, Leuven, 1995).

Kirikkale University,Faculty of Sciences and Arts, Department of Mathematics, 71450 , KIRIKKALE, TURKEY.

E-mail address: kilarslan@yahoo.com

University of Kragujevac, Faculty of Science, Department of Mathematics and InFormatics, 34000, Kragujevac, Serbia.

E-mail address: nesovickg@sbb.rs 